\title{
A steady approach
}

\author{
From the discovery of the Seebeck effect in the nineteenth century to its application in the latest space probes, \\ thermoelectrics have carved out a niche for reliable applications.
}

te was the so-called seven minutes of terror. The NASA Perseverance rover successfully completed atmospheric entry and a controlled soft landing on Mars. Its mission is to use its large suite of tools to assess not only the past habitability of the Jezero Crater but also to select which mineral samples to cache for a future sample-return mission. This will require a sizable and stable energy source for a long-range, long-duration drive, and this energy supply is provided by the heat emitted by $\mathrm{PuO}_{2}$ pellets during radioactive decay in a radioisotope thermoelectric generator. This uses a thermocouple, composed of two different thermoelectric materials, to generate voltage. The usage of thermoelectrics in space exploration is probably the highest-profile application of these materials, which rely on an effect that was first observed in the nineteenth century.

The discovery of thermoelectricity is commonly associated with Thomas Seebeck's observation in 1821 that heating a metallic junction could deflect a magnetic compass needle, although it was the work of Hans Ørsted in 1823 that noted generation of electric currents and coined the term thermoelectricity ${ }^{1}$. The basis of thermoelectric power generation is the Seebeck effect, where a gradient in temperature along a material can generate a voltage differential, with the Seebeck coefficient (or thermopower) controlling the size of this voltage difference: the larger the Seebeck coefficient the greater the induced voltage. In longitudinal thermoelectric materials both the voltage and temperature gradients run along the same axis. The decoupling of electrical conductivity (which should be large for efficient power generation) and thermal conductivity (which should be small to maintain the temperature differential) represents a significant materials and device design problem. An alternative would be to generate thermoelectricity using a 'transverse' approach, where the temperature and voltage gradients are perpendicular, such as in the Nernst effect, simplifying device design ${ }^{2}$. However, the Nernst thermopower is typically much smaller than the equivalent Seebeck coefficient, limiting transverse thermoelectric applications.

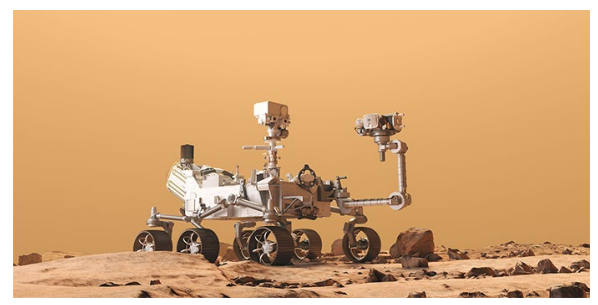

An artist's impression of the Perseverance rover on Mars. Credit: Neko / Alamy Stock Photo

In this issue, a Letter by Yuya Sakuraba and colleagues outlines an alternative methodology for large transverse thermopower. They make a closed circuit composed of two materials, doped silicon (a thermoelectric), and $\mathrm{Co}_{2} \mathrm{MnGa}$ (a magnetic material). Upon application of heat the thermoelectric generates a current, which then flows through the magnetic material. The current driven through $\mathrm{Co}_{2} \mathrm{MnGa}$ generates a transverse voltage, but via the anomalous Hall effect (AHE) that exists in the absence of an applied magnetic field. This leads to a transverse thermopower of $82.3 \mu \mathrm{V} \mathrm{K} \mathrm{K}^{-1}$, an order of magnitude larger than other transverse thermopowers. The researchers also present a phenomenological theory for the prediction of the transverse voltage in their device, which, as noted by Andrew May and Brian Sales in their News \& Views, can be used to look for better material combinations: researchers should search for materials with a large AHE. May and Sales also propose a simpler hybrid geometry with a single large-area thermoelectric leg that reduces compatibility issues, and note that although the voltage generated is less than that generated by longitudinal devices, the ease of implementation in power generation could be a significant advantage.

Thermoelectrics have been commercialized for several decades as temperatures sensors, but also as small-scale coolers where the heat load is $25 \mathrm{~W}$ or less and they can out-compete vapour compression cooling. This is due to another aspect of thermoelectricity, the Peltier effect. As observed by Jean Peltier in 1834, the passage of electrical current through a junction of two materials can result in heating or cooling. A Perspective by Zhifeng Ren and colleagues discusses recent progress in materials for thermoelectric cooling. In comparison with thermoelectric power generation, which relies on a large temperature gradient with the hot side several hundred kelvin hotter than the cool side, thermoelectric coolers are typically used near ambient temperature, reducing thermal stress on the thermocouple. The researchers note that the low-temperature thermoelectric properties of many materials are yet to be comprehensively investigated and so have been overlooked for cooling applications. They also note that a large mobility difference ratio between electrons and holes is important for generating large Seebeck coefficients, which are also relevant for larger cooling power. This can compensate for the bipolar conduction inherent in very narrow semiconductors, and suggests that exploration of these materials as well as semimetals could be a promising strategy for the development of better low-temperature thermoelectrics.

To have a larger impact for everyday life on Earth, research on thermoelectrics is being pursued on several fronts. Typically, the best room-temperature inorganic thermoelectrics are $\mathrm{Bi}_{2} \mathrm{Te}_{3}$ alloys, but the high cost of tellurium can limit applications, and thus researchers have considered alternatives such as $\mathrm{Mg}_{3} \mathrm{BiSb}$ alloys ${ }^{3,4}$. Typically, thermoelectric materials are brittle, whereas better flexibility could allow close contact between device and heat source ${ }^{5}$. And there is an ongoing search to improve figures of merit for power generation, for applications such as heat harvesting at the microscale or in solar thermoelectric generators ${ }^{6}$. With the current need for improving energy efficiency, the usage of these materials for waste heat harvesting or energy-efficient coolers will surely continue.

Published online: 26 March 2021 https://doi.org/10.1038/s41563-021-00982-9

\footnotetext{
References

1. Beretta, D. et al. Mat. Sci. Eng. R 138, 100501 (2019).

2. Sakai, A. et al. Nature 581, 53-57 (2020).

3. Zhang, J. et al. Nat. Commun. 8, 13901 (2017).

4. Mao, J. et al. Science 365, 495-498 (2019).

5. Jin, Q. et al. Nat. Mater. 18, 62-68 (2019).

6. Kraemer, D. et al. Nat. Mater. 10, 532-538 (2011).
} 